\title{
Supplementary material for Modelling steady states and the transient response of debris-covered glaciers
}

J. Ferguson and A. Vieli 

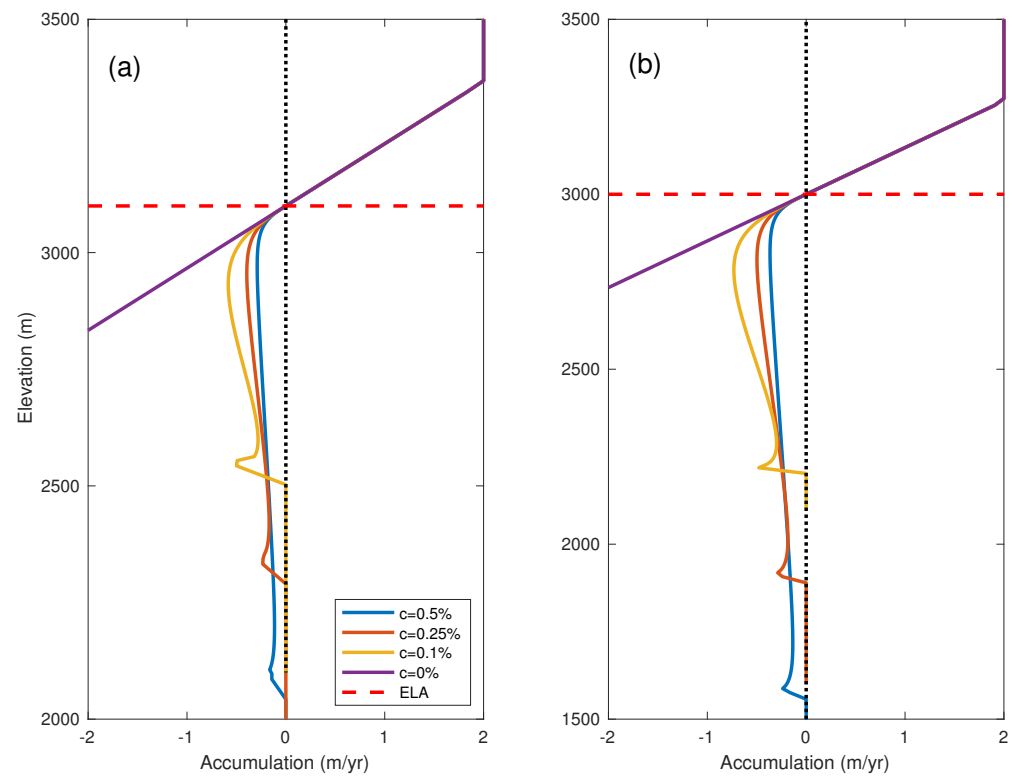

Figure S1: Surface mass balance profiles for steady state glaciers at (a) ELA = $3100 \mathrm{~m}$ and (b) ELA $=3000 \mathrm{~m}$ for different debris concentrations, corresponding to Fig. 1 in the main paper. 

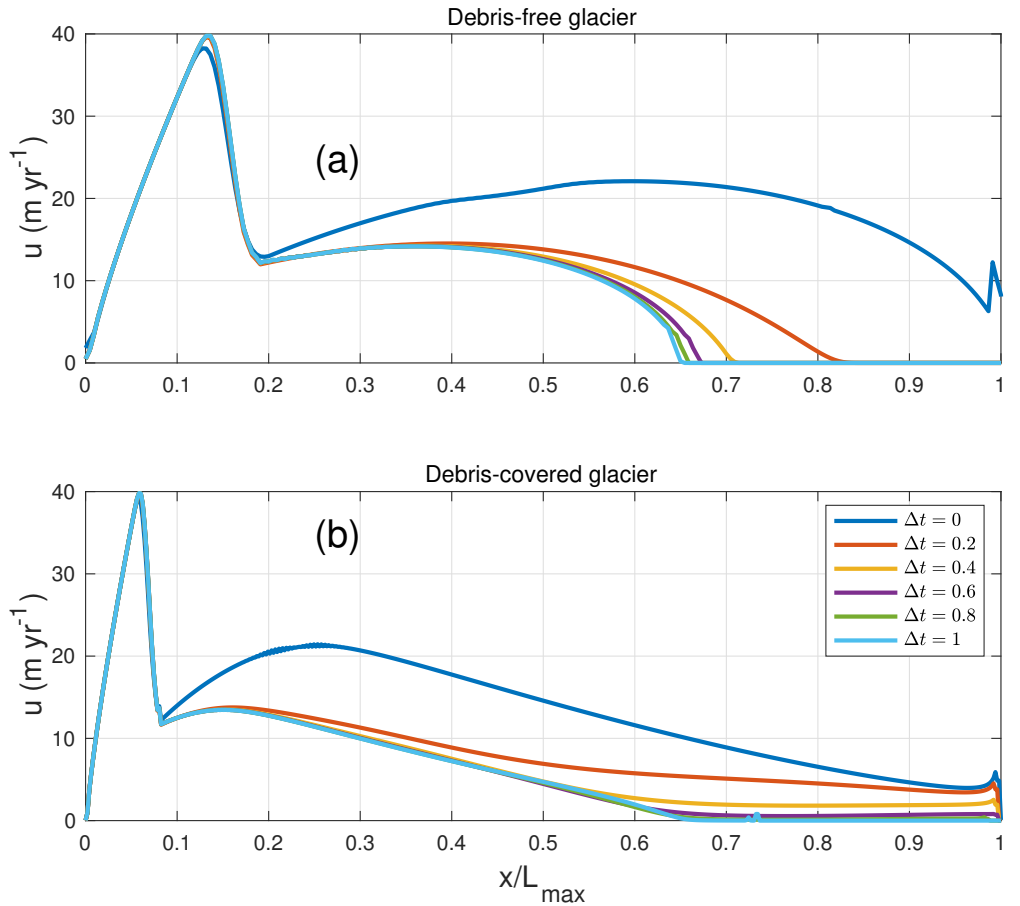

Figure S2: Velocity profiles for (a) a debris-free glacier and (b) a debris-covered glacier with $c=0.25 \%$ at different times during a transient retreat. The different coloured lines refer to the time relative to the time it takes to retreat to steady state, where a time of $t=0.1$ corresponds in the debris-free case to about 38 years and in the debris-covered case to about 40 years. This corresponds to Fig. 4 in the main paper. 

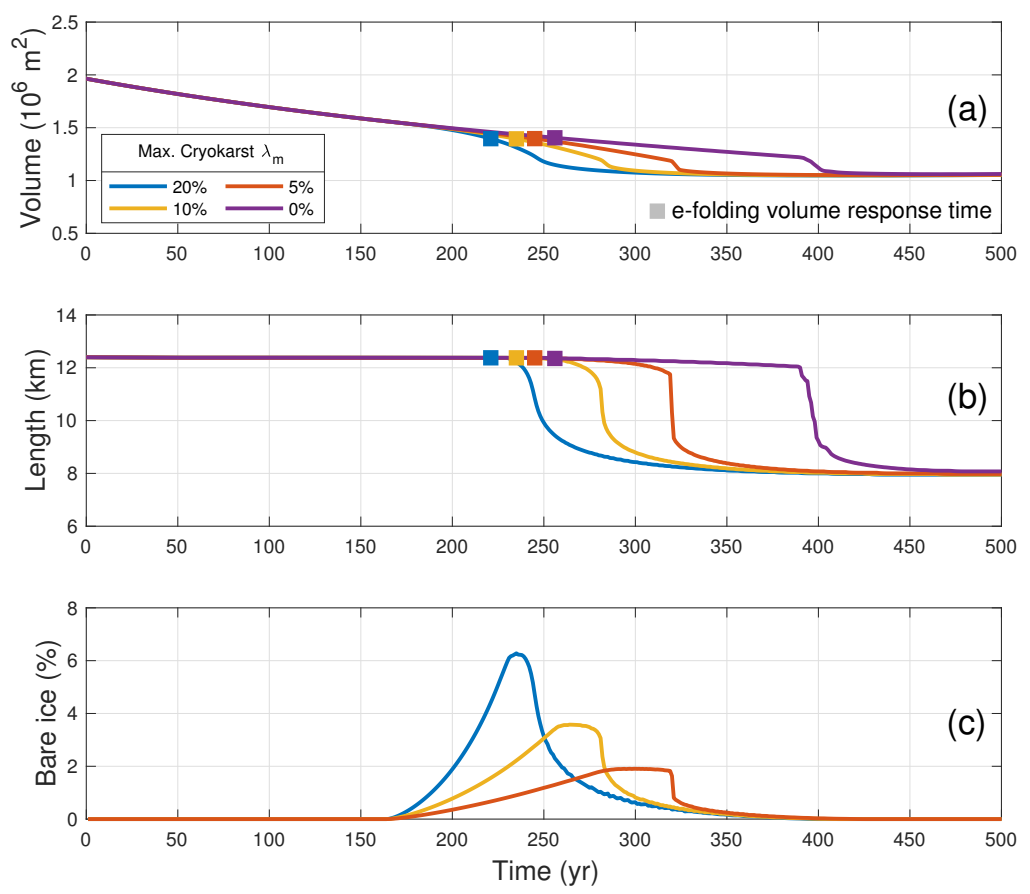

Figure S3: Transient volume response (a) and length response (b) for debriscovered glaciers with terminal cryokarst features retreating from steady state after a $100 \mathrm{~m}$ step change in ELA. Each colour represents a different value of the maximum cryokarst percentage $\lambda_{m}$. In all cases, the debris concentration is $c=0.25 \%$ and the driving stress thresholds are $\tau_{d}^{+}=100 \mathrm{kPa}$ and $\tau_{d}^{-}=50 \mathrm{kPa}$. The filled in squares in (a) and (b) represent the $e$-folding volume response time. The percentage of total debris-covered length that has a bare ice equivalent surface mass balance due to the presence of cryokarst is shown in (c). Compare with Fig. S4 below and Fig. 6 in the main paper. 

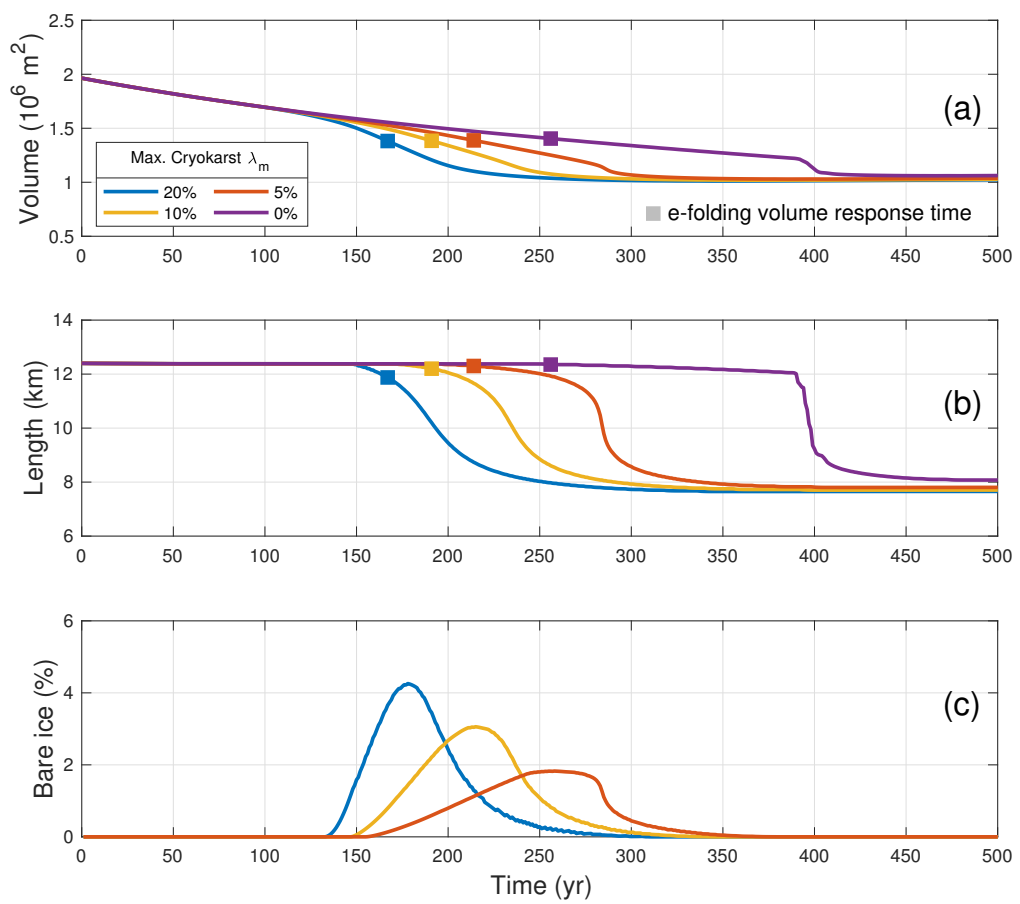

Figure S4: Transient volume response (a) and length response (b) for debriscovered glaciers with terminal cryokarst features retreating from steady state after a $100 \mathrm{~m}$ step change in ELA. Each colour represents a different value of the maximum cryokarst percentage $\lambda_{m}$. In all cases, the debris concentration is $c=0.25 \%$ and the driving stress thresholds are $\tau_{d}^{+}=125 \mathrm{kPa}$ and $\tau_{d}^{-}=75 \mathrm{kPa}$. The filled in squares in (a) and (b) represent the $e$-folding volume response time. The percentage of total debris-covered length that has a bare ice equivalent surface mass balance due to the presence of cryokarst is shown in (c). Compare with Fig. S3 above and Fig. 6 in the main paper. 

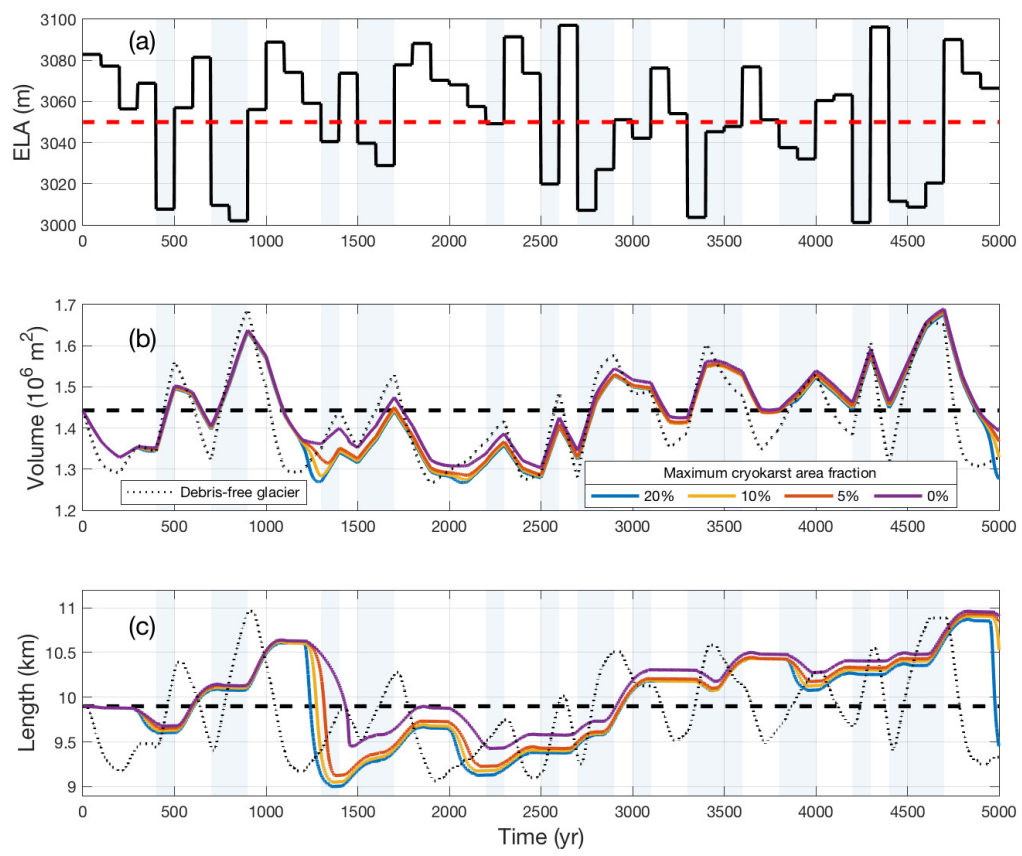

Figure S5: White noise climate forcing (a) and the corresponding transient volume response (b) and transient length response (c) for glaciers of different maximum cryokarst fractions $\lambda_{m}$. The dashed lines represent the mean value climate of ELA $=3050 \mathrm{~m}$ (a) and the corresponding steady state volume (b) and length (c). The light blue background shading represents temporal periods during which the climate forcing is colder than the mean climate. In all cases, the debris concentration is $c=0.25 \%$ and the cryokarst driving stress thresholds are $\tau_{d}^{+}=100 \mathrm{kPa}$ and $\tau_{d}^{-}=50 \mathrm{kPa}$. Compare with Fig. S6 below and Fig. 7 in the main paper. 

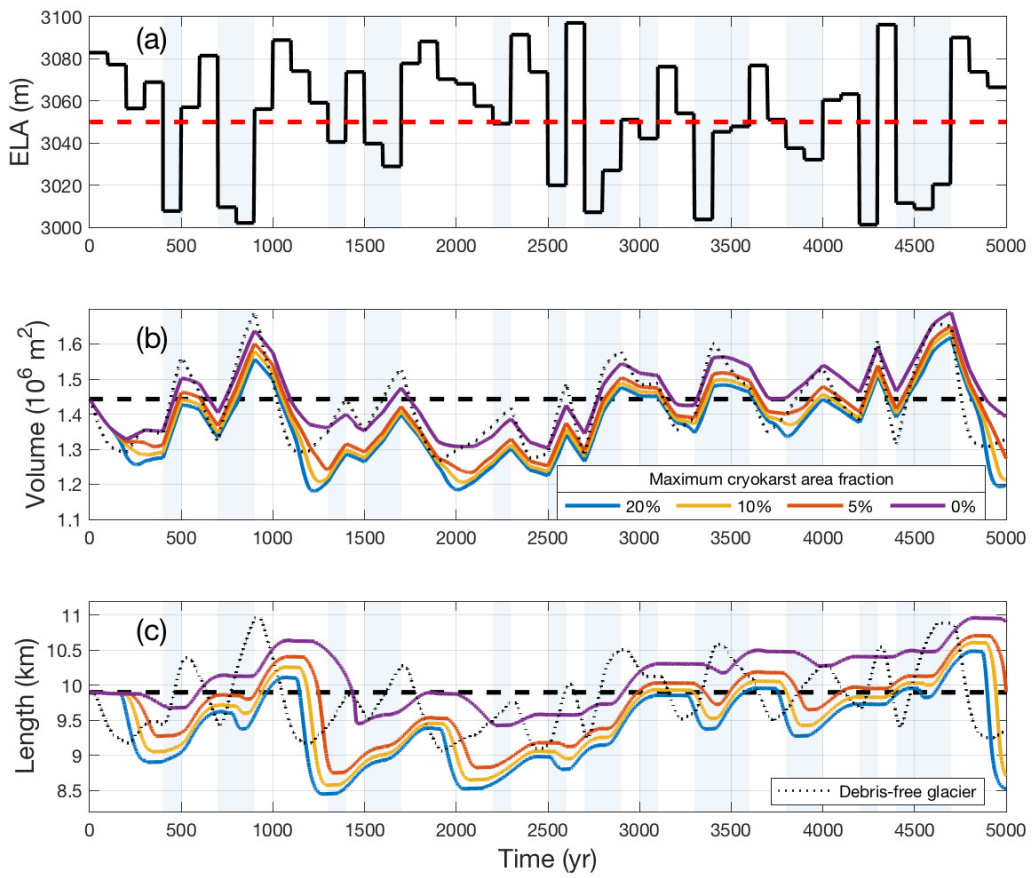

Figure S6: White noise climate forcing (a) and the corresponding transient volume response (b) and transient length response (c) for glaciers of different maximum cryokarst fraction $\lambda_{m}$. The dashed lines represent the mean value climate of ELA $=3050 \mathrm{~m}$ (a) and the corresponding steady state volume (b) and length (c). The light blue background shading represents temporal periods during which the climate forcing is colder than the mean climate. In all cases, the debris concentration is $c=0.25 \%$ and the cryokarst driving stress thresholds are $\tau_{d}^{+}=125 \mathrm{kPa}$ and $\tau_{d}^{-}=75 \mathrm{kPa}$. Compare with Fig. S5 above and Fig. 7 in the main paper. 